\title{
PVT1 regulates inflammation and cardiac function via the MAPK/NF-кB pathway in a sepsis model
}

\author{
FANG FENG, YAN QI, CHENMING DONG and CHAOHUI YANG \\ Department of Critical Care Medicine, The Second Hospital of Lanzhou University, Lanzhou, Gansu 730000, P.R. China
}

Received May 30, 2018; Accepted August 22, 2018

DOI: $10.3892 / \mathrm{etm} .2018 .6814$

\begin{abstract}
The aim of the present study was to investigate the role of plasmacytoma variant translocation gene 1 (PVT1) in the occurrence and development of sepsis-induced inflammation and cardiac dysfunction and its underlying mechanism. A sepsis rat model was first established by cecal ligation and puncture. The mRNA levels of PVT1 and microRNA-143 in the myocardial tissues of rats were detected by reverse transcription-quantitative polymerase chain reaction (RT-qPCR) analysis. Cardiac function, levels of myocardial injury markers and inflammatory indicators were detected following PVT1 knockdown. The regulatory effect of microRNA-143 on PVT1 was assessed using a luciferase reporter gene assay and RT-qPCR analysis. The specific role of PVT1 in regulating the mitogen-activated protein kinase $(\mathrm{MAPK}) /$ nuclear factor $(\mathrm{NF})-\kappa \mathrm{B}$ pathway was detected using western blot analysis. PVT1 was downregulated and microRNA-143 was upregulated in the myocardial tissues of sepsis rats. The left ventricular peak pressure was markedly decreased in the sepsis rats. By contrast, the left ventricular end diastolic pressure, levels of inflammatory indicators, myocardial injury markers and complement proteins of $\mathrm{C} 5$ and $\mathrm{C} 5 \mathrm{a}$ were increased in the sepsis rats. The above changes were reversed by PVT1 knockdown or the upregulation of microRNA-143. MicroRNA-143 was confirmed as being bound to PVT1 using the luciferase reporter gene assay and RT-qPCR analysis. Upregulated PVT1 was capable of activating the MAPK/NF- $\mathrm{BB}$ pathway. Taken together, PVT1 was upregulated in the myocardial tissues of sepsis rats, which inhibited cardiac function and promoted the secretion of inflammatory factors; and the mechanism was associated

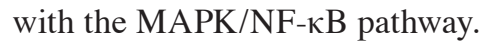

Correspondence to: Dr Fang Feng, Department of Critical Care Medicine, The Second Hospital of Lanzhou University, 82 Cuiying Gate, Chengguan, Lanzhou, Gansu 730000, P.R. China

E-mail: emzo00@163.com

Key words: plasmacytoma variant translocation gene 1, mitogen-activated protein kinase/nuclear factor- $\kappa \mathrm{B}$ pathway, sepsis, cardiac function, inflammation

\section{Introduction}

Sepsis is the leading cause of infection complications. Sepsis-induced multiple organ dysfunction syndrome severely threatens the health of infected patients with a mortality rate of $33.3 \%$ worldwide (1). A large number of clinical studies have shown that patients with sepsis experience fever, shock, and can succumb to mortality due to excessive inflammatory reactions. A sepsis animal model induced by injection of tumor necrosis factor (TNF)- $\alpha$ or interleukin (IL)-1 has also confirmed the dangers of sepsis to the fetus. Myocardial injury is frequently observed in patients with sepsis, with a prevalence of up to $40 \%$ (2). Although adequate fluid resuscitation and vasoactive drugs are immediately applied, decompensated circulatory failure remains a significant challenge in clinical treatment (3). The excessive release of a large number of cytokines and inflammatory mediators seriously damages cardiac function in patients with sepsis $(4,5)$.

With the extensive use of RNA-sequencing technology, comprehensive analysis of transcription has progressed substantially. Over $98 \%$ of genomes do not encode proteins, which are termed non-coding RNAs (ncRNAs) (6). Long non-coding RNAs (lncRNAs) are a class of ncRNAs $>200 \mathrm{nt}$ in length. IncRNAs have temporospatial characteristics, and are incapable of encoding proteins as the meaningful open reading frame is lacking. Previous studies have revealed that lncRNAs are involved in the pathophysiology of tumors by transcriptional or post-transcriptional regulation, chromosomal rearrangement, endogenous small interfering RNA production, and the construction of protein complexes (7).

The transcript of the human gene plasmacytoma variant translocation gene 1 (PVT1) belongs to the intergenic lncRNAs. PVT1 is located in the sense strand of chromosome $8 \mathrm{q} 24$, which spans genome intervals for $>300 \mathrm{~kb}(8)$. In tumor cells, the chromosome 8q24 region is the highest susceptible target for DNA amplification. Its abnormal amplification indicates a high risk of tumor occurrence. In addition, chromosome 8q24 contains various risk sites that are associated with the terminal stage of type 2 diabetic nephropathy and non-integrative cleft lip and palate $(9,10)$. However, the specific role of PVT1 in myocardial diseases requires further investigation. The aim of the present study was to investigate the role of PVT1 in the occurrence and development of sepsis-induced inflammation and cardiac dysfunction and its underlying mechanism. 


\section{Materials and methods}

Animals. In total, 60 male rats of SPF grade (8 weeks old, weighing 180-220 g; Experimental Animals Center of Lanzhou University, Lanzhou, China) were selected for the present study. The rats were housed in a temperature-controlled room $\left(21 \pm 2^{\circ} \mathrm{C}\right)$ on a 12:12-h light/dark cycle (lights on at $\left.06: 00\right)$. All rats had free access to water and food.

Sepsis rat model construction. The sepsis model was constructed using the cecal ligation and puncture (CLP) procedure (11). Briefly, the rats were anesthetized with $2.5 \%$ pentobarbital $(40 \mathrm{mg} / \mathrm{kg})$ intraperitoneally and positioned on the operating table. A longitudinal midline incision was made to expose the abdominal cavity. The mesentery was carefully separated and colon contents were lightly squeezed to fill back to the cecum. It is important that the cecal branch of ileocecal artery is avoided to prevent hemorrhage. Subsequently, $30 \%$ of the cecum was ligated and punctured with an 18-gauge needle between the ligation and terminal. The cecum was returned to the abdomen and the incision was sutured. The rats were housed in the feeding room at $22^{\circ} \mathrm{C}$ following CLP. Vital signs were observed until revival from anesthesia and every $6 \mathrm{~h}$ subsequently. The postoperative rats were given free access to food and water without any other treatments. A similar surgical procedure was performed in the sham group, with the exception of the ligation and puncture of the cecum. The rats used in this experiment were obtained from the Model Animal Research Center of Nanjing University (Nanjing, China). All experiments were approved by the Animal Ethics Committee of Lanzhou University (Lanzhou, China).

Lentivirus transfection. The rats in the experimental group were depressed following the surgical procedure, the food and water intake was reduced, and there was a lag in response. The rats of the same age were randomly assigned into the sham group ( $\mathrm{n}=10)$, CLP group ( $\mathrm{n}=10), \mathrm{CLP}+$ lentivirus (LV)-vector group $(\mathrm{n}=10), \mathrm{CLP}+\mathrm{LV}$-short hairpin (sh)PVT1 group $(\mathrm{n}=10)$, CLP + microRNA-NC group $(\mathrm{n}=10)$ and CLP + microRNA-143 mimics group $(n=10)$. The oligonucleotide sequences and lentiviruses used in the experiments were all constructed by GenePharma (Shanghai, China). Sequences for shPVT1 were as follows: shPVT1 \#1 forward, 5'-GCUUGGAGGCUGAGG AGUUTT-3' and reverse, 5'-AACUCCUCAGCCUCCAAG CTT-3'; shPVT1 \#2 forward, 5'-CCCAACAGGAGGACA GCUUTT-3' and reverse, 5'-AAGCUGUCCUCCUGUUGG GTT-3'. The sequence for microRNA-NC was 5'-UUCUCC GAACGUGUCACGUTT-3'. The sequence for microRNA-143 mimics was 5'-UGAGAUGAAGCACUGUAGCUC-3'. The corresponding lentivirus (multiplicity of infection=20), $100 \mathrm{nM}$ microRNA-NC or $100 \mathrm{nM}$ microRNA-143 mimic was administrated into the tail vein following surgery. Each rat was housed in an individual cage following revival from anesthesia. Serum samples were obtained from the tail vein $24 \mathrm{~h}$ following surgery. A limulus assay (Bioendo Technology, Xiamen, China) was used to detect endotoxin concentrations. The rats were anaesthetized with $10 \%$ chloral hydrate $(400 \mathrm{mg} / \mathrm{kg}$, intraperitoneally; Beyotime Institute of Biotechnology, Shanghai, China) and then sacrificed by cervical dislocation $24 \mathrm{~h}$ after surgery and myocardial tissues were harvested and preserved in liquid nitrogen for the following experiments. No rats exhibited signs of peritonitis following the administration of $10 \%$ chloral hydrate.

Cell culture and transfection. The H9c2 rat cardiomyocyte cell line was obtained from American Type Culture Collection (Manassas, VA, USA). The H9c2 cells were cultured at $37^{\circ} \mathrm{C}$ with 5\% $\mathrm{CO}_{2}$ in Dulbecco's modified Eagle's medium supplemented with $10 \%$ fetal bovine serum (both Gibco; Thermo Fisher Scientific, Inc., Waltham, MA, USA), $100 \mathrm{IU} / \mathrm{ml}$ penicillin and $10 \mu \mathrm{g} / \mathrm{ml}$ streptomycin. Cell transfection was performed using Lipofectamine 2000 (Invitrogen; Thermo Fisher Scientific, Inc.) according to the manufacturer's protocol when the confluence was up to $70-80 \%$. The culture medium was replaced every $6 \mathrm{~h}$. The transfected cells were collected for the following in vitro experiments.

Cardiac function determination. Following anesthesia of the rats, left intraventricular intubation was performed via the right common carotid artery. The MFLab 3.01 package in the FDP-1 HRV \& BRS analysis system was used to monitor left ventricular peak pressure (LVPP) and left ventricular end diastolic pressure (LVEDP).

Enzyme-linked immunosorbent assay (ELISA). The corresponding reagents were prepared and placed at room temperature. The sample or standard solution $(100 \mu \mathrm{l})$ was added to sample wells or standard wells, respectively. CK-MB (cat. no. ab31832), cTnI (cat. no. ab47003), C5 (1:200, ab4616), C5a (cat. no. ab194637), TNFa (cat. no. ab220210), IL-5 (cat. no. ab200248), IL-1 $\beta$ (cat. no. ab46608), IL-10 (cat. no. ab100765), IL-17 (cat. no. ab214028) and IFN $\gamma$ (cat. no. ab46107; all 1:200; Abcam, Cambridge, MA, USA) primary antibodies were then added for incubation at room temperature for a total of $2 \mathrm{~h}$. Subsequently, horseradish peroxidase-labeled antibodies were added. Following incubation for $1 \mathrm{~h}$ at room temperature, $100 \mu \mathrm{l}$ of a substrate (a mixture of solutions A and B) was added in the dark for $10 \mathrm{~min}$ to get a color reaction. The absorbance value at the wavelength of $450 \mathrm{~nm}$ was detected using a microplate reader (Bio-Rad Laboratories, Inc., Hercules, CA, USA).

Luciferase reporter gene assay. The Kyoto Encyclopedia of Genes and Genomes (KEGG) database (https://www.kegg. $\mathrm{jp} /$ ) was used to predict the upstream gene of PVT1. The H9c2 cells were co-transfected with wild-type PVT1 (PVT1-WT) or mutant-type PVT1 (PVT1-MUT) and microRNA-negative control (NC) or microRNA-143 mimics (all Tiangen Biotech Co., Ltd., Beijing, China), respectively. The pRL-TK vector was transfected as the loading control. The luciferase activity was determined according to the instructions of the relative commercial kit. The relative luciferase activity was determined as the luciferase activity of firefly/luciferase activity of Renilla.

$R N A$ extraction and reverse transcription-quantitative polymerase chain reaction ( $R T-q P C R)$ analysis. A TRIzol kit (Invitrogen; Thermo Fisher Scientific, Inc.) was used to extract total RNA from the tissues. The reverse transcription 

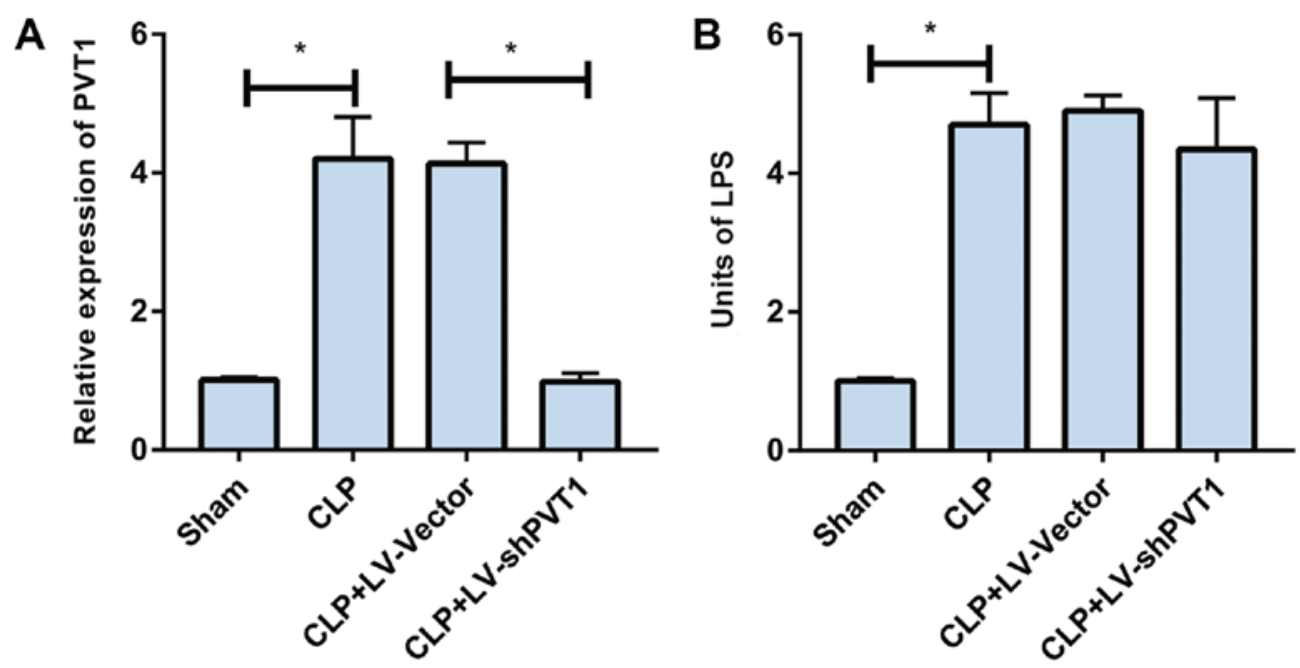

Figure 1. PVT1 is upregulated in myocardial tissues of sepsis rats. PVT1 was examined by reverse transcription-quantitative polymerase chain reaction analysis. (A) The expression of PVT1 and (B) the serum level of endotoxin in the sham group, CLP group, CLP + LV-vector group and CLP + LV-shPVT1 group. PVT1, plasmacytoma variant translocation gene 1; CLP, cecal ligation and puncture; LV, lentivirus; sh, short hairpin RNA. "P<0.05.

reaction was performed based on the manufacturer's protocol of the PrimeScript RT reagent kit (Takara Bio, Inc., Tokyo, Japan). The obtained complementary cDNA was amplified by the following PCR amplification reaction conditions: Denaturation at $94^{\circ} \mathrm{C}$ for $30 \mathrm{sec}$, annealing at $55^{\circ} \mathrm{C}$ for $30 \mathrm{sec}$ and extension at $72^{\circ} \mathrm{C}$ for $90 \mathrm{sec}$, for a total of 40 cycles. Reverse transcription system was $20 \mu \mathrm{l}$ : $1 \mu \mathrm{l}$ Total RNA, $5 \mu \mathrm{l}$ 5x Transcript All-in-One SuperMix for qPCR, $2.5 \mu \mathrm{lgDNA}$ Remover, $0.5 \mu \mathrm{l}(10 \mu \mathrm{M})$ for each primer and RNase-free water (7). The primers used in the experiment were as follows: PVT1 forward, 5'-GGGGTACCCTCCGGGCAGAGCGCG TGTG-3' and reverse, 5'-CGGGATCCTAGACACGAGGCC GGCCACGC-3'; GAPDH forward, 5'-ACCCACTCCTCC ACCTTTGA-3' and reverse, 5'-CTGTTGCTGTAGCCAAAT TCGT-3'. Relative quantitative analysis of data was carried out by $2^{-\Delta \Delta \mathrm{Cq}}$ method (12).

Western blot analysis. Total protein was extracted from the treated cells using radioimmunoprecipitation assay solution (Beyotime Institute of Biotechnology). The bicinchoninic acid method was used for quantitative protein concentration. The protein sample (10 $\mu \mathrm{l} / \mathrm{lane})$ was separated by electrophoresis on a $10 \%$ sodium dodecyl sulfate-polyacrylamide gel and then transferred onto a polyvinylidene fluoride membrane (EMD Millipore, Billerica, MA, USA). The membranes were blocked with skimmed milk, following which the membranes were incubated with primary antibodies, including p-p38 (cat. no. 4511), p38 (cat. no. 8690),

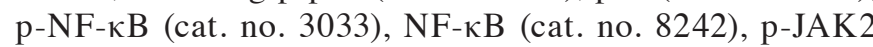
(cat. no. 3776), JAK2 (cat. no. 4040), p-STAT3 (cat. no. 9145), STAT3 (cat. no. 9139), GAPDH (cat. no. 2118; all 1:500; Cell Signaling Technology, Inc., Danvers, MA, USA) overnight at $4^{\circ} \mathrm{C}$. The membranes were then washed with Tris-buffered saline with Tween-20, followed by incubation with horseradish peroxidase-conjugated goat anti-rabbit IgG secondary antibodies (cat. no. ab6721; 1:2,000; Abcam). The protein blot on the membrane was exposed by an enhanced chemiluminescence (ECL) kit (EMD Millipore). The gray value by ImageJ (version 1.38; National Institutes of Health,
Bethesda, MA, USA) is used for the densitometric analysis of protein.

Statistical analysis. All statistical analyses were performed using Statistical Product and Service Solutions (SPSS) 17.0 software (SPSS, Inc., Chicago, IL, USA). GraphPad Prism 5 (GraphPad Software, Inc., La Jolla, CA, USA) was introduced for image editing. Measurement data are expressed as the mean \pm standard deviation. An independent-sample t-test was used to compare the differences between two groups. One-way analysis of variance followed by a post hoc test (Scheffé's method) was applied for multiple comparisons of groups. $\mathrm{P}<0.05$ was considered to indicate a statistically significant difference.

\section{Results}

PVT1 is upregulated in myocardial tissues of sepsis rats. Initially, the rat sepsis model was successfully established by CLP. PVT1 was upregulated in the myocardial tissues of the sepsis rats, as demonstrated by RT-qPCR analysis. The LV-vector and LV-shPVT1 vectors were constructed and injected into the sepsis rats via the tail vein, respectively. PVT1 was markedly lower in the rats injected with LV-shPVT1 than in those injected with the LV-vector (Fig. 1A). Subsequently, the serum levels of endotoxin in rat myocardial tissues were detected. The data indicated that the endotoxin level was markedly increased in the sepsis rats. LV-shPVT1 injection did not affect the endotoxin level in the sepsis rats (Fig. 1B).

PVT1 regulates sepsis-induced inflammation and cardiac function. Lower LVPP and decreased LVEDP were observed in the rats of the CLP group than those of the sham group, which were reversed by LV-shPVT1 injection (Fig. 2A and B). Subsequently, the serum levels of myocardial injury markers were detected in each rat. The data demonstrated that the levels of creatine kinase (CK), CK-MB and cardiac troponin I (cTn-I) were markedly higher in the rats of the CLP group than in those of the sham group, which were also 

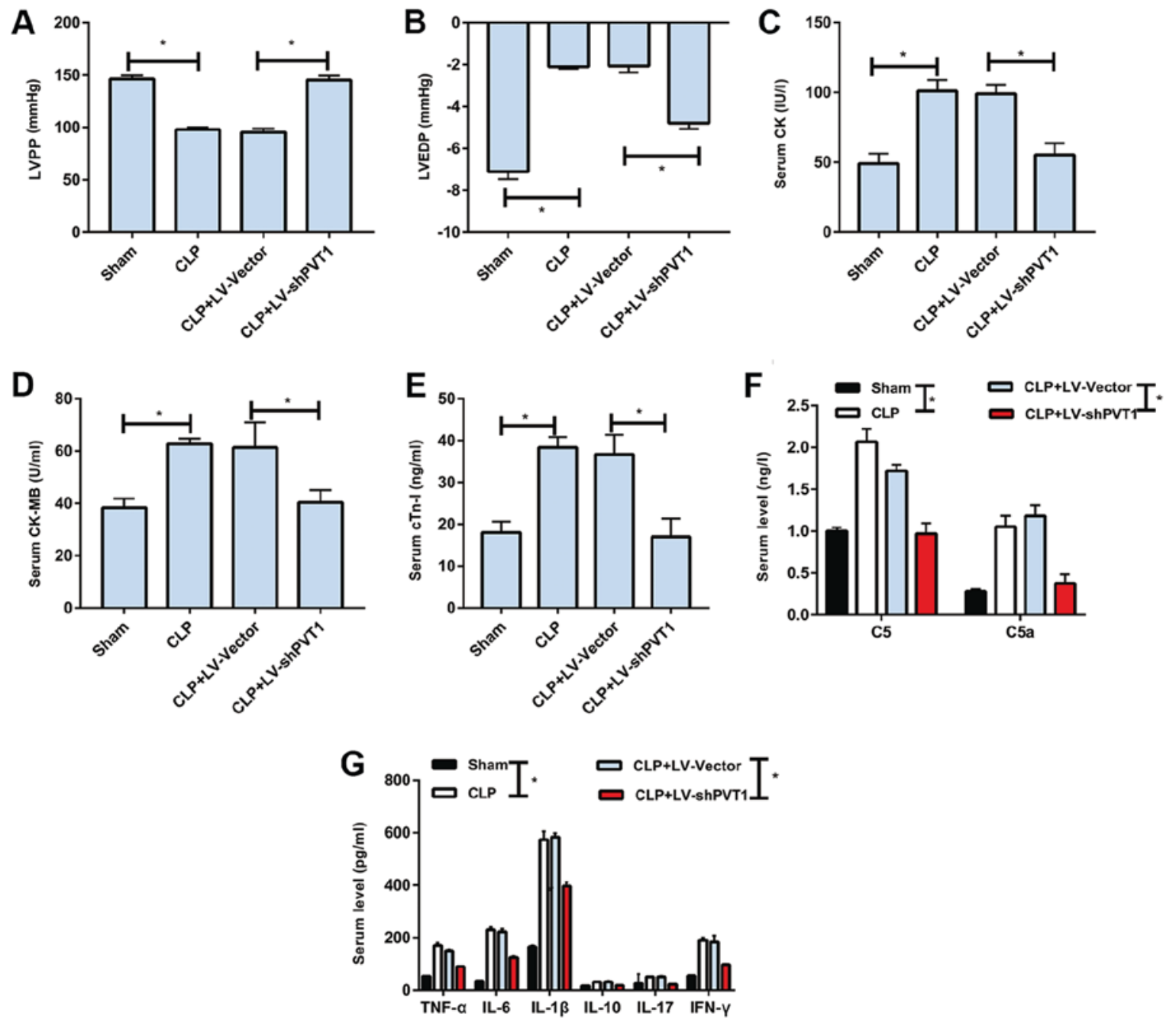

Figure 2. PVT1 regulates sepsis-induced inflammation and cardiac function. (A) LVPP, (B) LVEDP, (C) CK, (D) CK-MB and (E) cTn-I levels in the sham group, CLP group, CLP + LV-vector group and CLP + LV-shPVT1 group. Serum levels of (F) C5, C5a, (G) TNF- $\alpha$, IL-1 $\beta$, IL-6, IL-10, IL-17 and IFN- $\gamma$ in the sham group, CLP group, CLP + LV-vector group and CLP + LV-shPVT1 group. PVT1, plasmacytoma variant translocation gene 1; CLP, cecal ligation and puncture; LV, lentivirus; sh, short hairpin RNA; LVPP, left ventricular peak pressure; LVEDP, left ventricular end diastolic pressure; CK, creatine kinase; cTn-I, cardiac troponin I; TNF- $\alpha$, tumor necrosis factor- $\alpha$; IL, interleukin; IFN- $\gamma$, interferon- $\gamma$. "P<0.05.
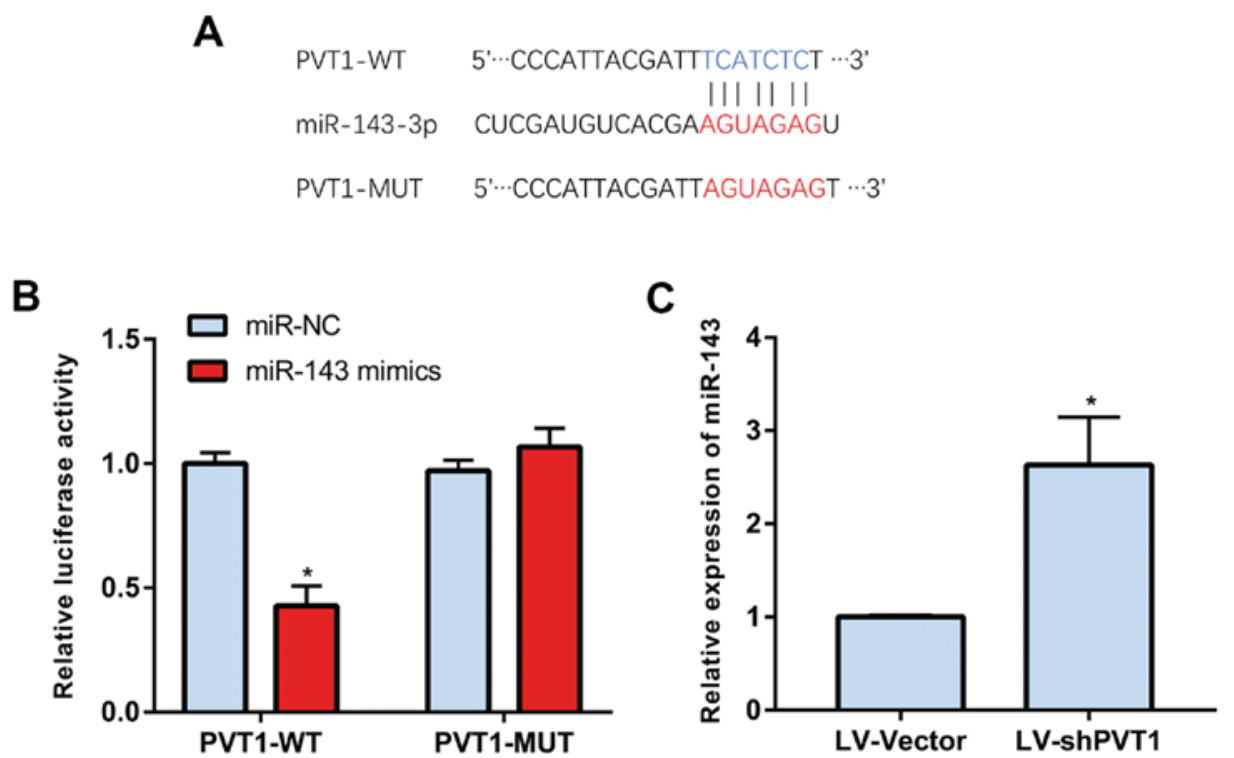

Figure 3. PVT1 is regulated by miR-143. (A) Binding site of PVT1 and microRNA-143. (B) miR-143 was found to directly bind to PVT1-WT. (C) Expression of microRNA-143 following PVT1 knockdown. PVT1, plasmacytoma variant translocation gene 1; miR, microRNA; NC, negative control; LV, lentivirus; sh, short hairpin RNA; WT, wild-type; MUT, mutant. "P<0.05. 
A

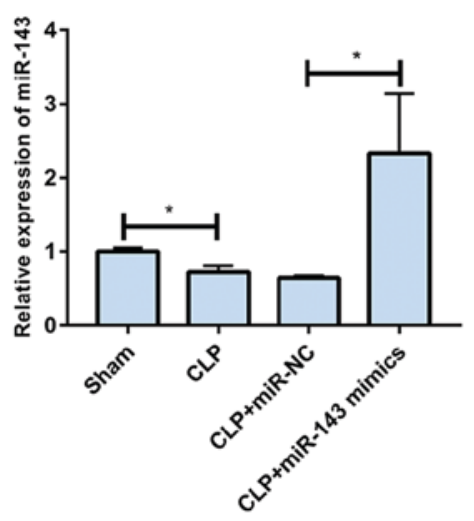

D

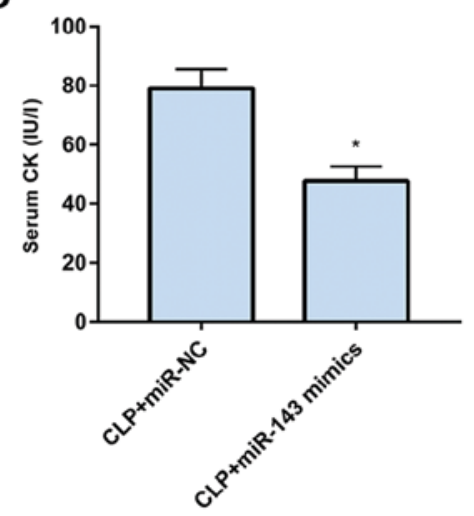

B
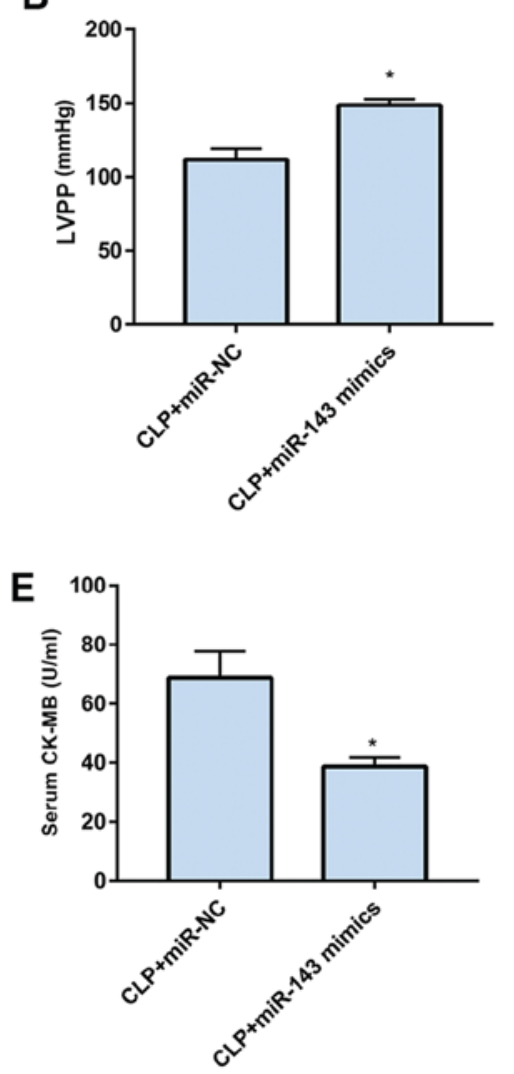

C
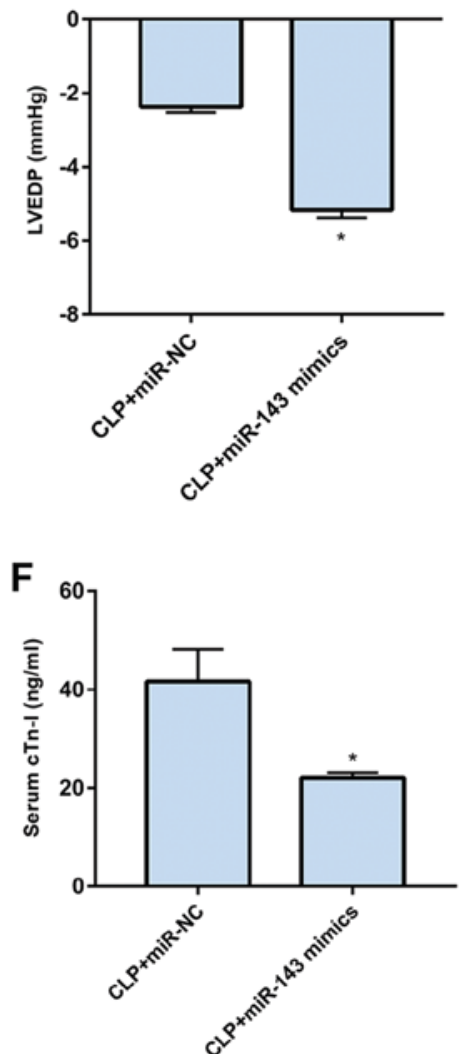

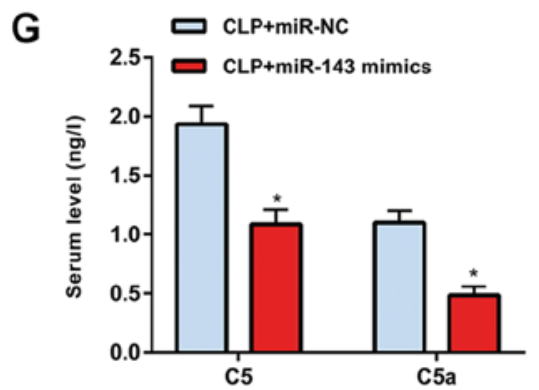

H

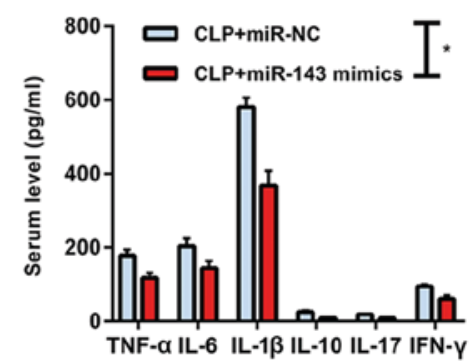

Figure 4. Overexpression of miR-143 improves sepsis-induced inflammation and cardiac function. (A) Levels of miR-143 in the sham group, CLP group, CLP + miR-NC group and CLP + miR-143 mimics group. (B) LVPP, (C) LVEDP, (D) CK, (E) CK-MB and (F) cTn-I levels in the CLP + miR-NC group and CLP + miR-143 mimics group. Serum levels of (G) C5, C5a, (H) TNF- $\alpha$, IL-1 $\beta$, IL-6, IL-10, IL-17 and IFN- $\gamma$ in the CLP + miR-NC group and CLP + miR-143 mimics group. PVT1, plasmacytoma variant translocation gene 1; CLP, cecal ligation and puncture; miR, microRNA; NC, negative control; LVPP, left ventricular peak pressure; LVRFP, left ventricular end diastolic pressure; CK, creatine kinase; cTn-I, cardiac troponin I; TNF- $\alpha$, tumor necrosis factor- $\alpha$; IL, interleukin; IFN- $\gamma$, interferon- $\gamma$. ${ }^{*} \mathrm{P}<0.05$.

reversed by LV-shPVT1 injection (Fig. 2C-E). Similarly, the complement proteins of $\mathrm{C} 5$ and $\mathrm{C} 5 \mathrm{a}$ were higher in the rats of the CLP group than in those of the sham group. LV-shPVT1 injection markedly reversed the elevated levels of complement proteins (Fig. 2F). Furthermore, inflammatory factors were found to be higher in serum samples from the CLR group in than those from sham group, including TNF- $\alpha$, IL-1 $\beta$, IL-6, IL-10, IL-17 and interferon (IFN)- $\gamma$. Following injection with LV-shPVT1, the elevated levels of inflammatory factors were markedly decreased (Fig. 2G). The above data demonstrated that PVT1 regulated sepsis-induced inflammation and cardiac function.

PVT1 is regulated by microRNA-143. PVT1 was predicted to be regulated by microRNA-143 through a bioinformatics method (Fig. 3A). The luciferase reporter gene assay revealed that the luciferase activity of PVT1-WT was markedly reduced, indicating that PVT1 was able to directly bind to microRNA-143 (Fig. 3B and C). These results confirmed that PVT1 may be regulated by microRNA-143.

MicroRNA-143 regulates sepsis-induced inflammation and cardiac function. The level of microRNA-143 was lower in rats of the CLP group than in those of the sham group, which was markedly reversed by transfection with microRNA-143 mimics (Fig. 4A). It was found that LVPP was elevated and LVEDP was reduced following microRNA-143 mimics transfection in the sepsis rats (Fig. 4B and C). In addition, the upregulation of microRNA-143 markedly decreased serum levels of CK, CK-MB and cTn-I in the sepsis rats (Fig. 4D-F). 


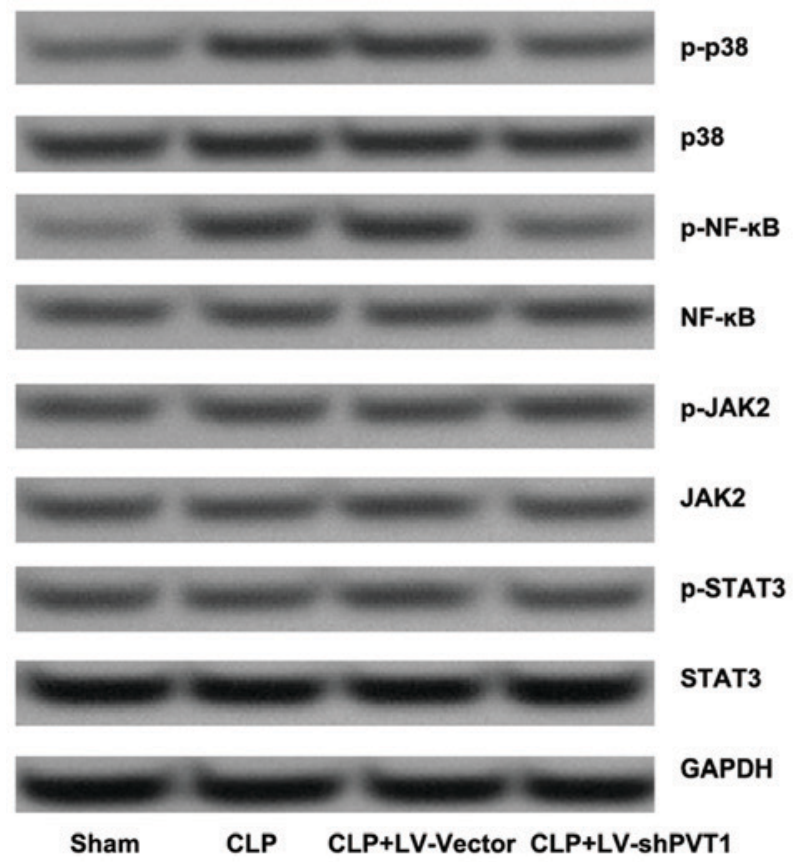

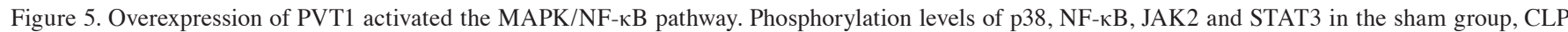
group, CLP + LV-vector group and CLP + LV-shPVT1 group. PVT1, plasmacytoma variant translocation gene 1; CLP, cecal ligation and puncture; LV, lentivirus; sh, short hairpin RNA; NF-кB, nuclear factor-кB; JAK2, Janus kinase 2; STAT3, signal transducer and activator of transcription; p-, phosphorylated. ${ }^{*} \mathrm{P}<0.05$.

The protein expression levels of C5 and C5a in the sepsis rats were also reduced following microRNA-143 mimics transfection (Fig. 4G). Furthermore, the upregulation microRNA-143 markedly reduced the serum levels of inflammatory factors (Fig. 4H).

PVT1 regulates the $M A P K / N F-\kappa B$ pathway. It has been reported that the $\mathrm{MAPK} / \mathrm{NF}-\kappa \mathrm{B}$ pathway is crucial in the inflammatory response (9). Therefore, the present study examined whether PVT1 regulates inflammation and cardiac function via the MAPK/NF- $\kappa \mathrm{B}$ pathway. The western blot results showed that the $\mathrm{MAPK} / \mathrm{NF}-\kappa \mathrm{B}$ pathway was activated in the sepsis rats, which was inhibited by PVT1 knockdown. However, no significant changes were found in the protein levels of Janus kinase 2 (JAK2) or signal transducer and activator of transcription 3 (Fig. 5). These data suggested that PVT1 regulates sepsis-induced inflammation and cardiac function via the $\mathrm{MAPK} / \mathrm{NF}-\kappa \mathrm{B}$ pathway.

\section{Discussion}

Sepsis-induced myocardial dysfunction occurs in the early stage of sepsis. It gradually progresses into cardiac insufficiency if effective treatment is not performed, which is the leading cause of mortality in patients with non-cardiogenic heart diseases (13). Substantial cumulative evidence indicates that various calcium transporters and the myofilaments are involved in sepsis-induced myocardial pathology (14). The inflammatory reaction caused by sepsis impairs cardiac glucose metabolism, inducing myocardial dysfunction (15). Inflammatory cytokines, including TNF- $\alpha$ and IL-6, are also involved in sepsis-induced myocardial injury (16).

TNF- $\alpha$ was previously considered to be secreted by activated macrophages, however, studies have shown that TNF- $\alpha$ can also be secreted by sepsis-stimulated cardiomyocytes (17). In a canine model of septic shock, TNF- $\alpha$ induced myocardial dysfunction in a dose-dependent manner $(18,19)$. IL-1 is synthesized by monocytes, macrophages and neutrophils stimulated by TNFs. Functionally, IL-1 inhibits myocardial contractility by stimulating nitric oxide synthase. IL-6, another important pro-inflammatory cytokine, is also involved in the pathogenesis of sepsis. IL- 6 is a better predictor of sepsis than TNF- $\alpha$ due to its longer persistence in the circulation (20). In the present study, it was found that downregulated PVT1 markedly reduced the expression levels of inflammatory cytokines (TNF- $\alpha$, IL-1 $\beta$, IL-6, IL-10, IL-17, and IFN- $\gamma$ ), suggesting that PVT1 is involved in regulating inflammation. Although cytokines are likely to be crucial at the early-systolic phase, previous in vitro experiments have found that cardiac function continues to decline even when sepsis has recovered $(21,22)$. Therefore, myocardial inhibitors are not the only reason for sepsis-induced myocardial injury.

MAPKs belong to the serine/threonine protein kinase family. MAPKs are widely present in eukaryotic cells. Extracellular signals, including physical stress, growth factors, inflammatory factors and bacterial complexes, can activate MAPKs, thereby stimulating cellular responses (23). $\mathrm{NF}-\kappa \mathrm{B}$ is a downstream molecule of p38 MAPK, which belongs to a type of nucleoprotein factor. There are five subtypes of NF- $\mathrm{B}$, namely Rel (i.e., c-Rel), RelA (i.e., p65),

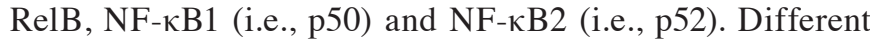
dimers can be formed with different subtypes, and the most common is p65/p50 (24,25). It has been shown that p65/p50 is closely associated with the activation of inflammatory genes induced by IL- $1 \beta$ or TNF- $\alpha$. The activation and inactivation of $\mathrm{NF}-\kappa \mathrm{B}$ are mainly regulated by the inhibitory subunit of $\mathrm{NF}-\kappa \mathrm{B}(\mathrm{I} \kappa \mathrm{B})$ and $\mathrm{I} \kappa \mathrm{B}-$ kinase $(\mathrm{IKK})$, respectively. The activation of $N F-\kappa B$ is strictly regulated; extracellular 
stimuli, including ischemia, hypoxia, calcium overload, oxygen free radicals and inflammatory factors, can activate IKK to phosphorylated (p-)IKK. Subsequently, p-IKK $\alpha$ and $\mathrm{p}-\mathrm{IKK} \beta$ phosphorylate serine residues of I $\mathrm{B} \boldsymbol{\alpha} \alpha$ (Ser32/Ser36) and serine residues of $\mathrm{I} \kappa \mathrm{B} \beta$ (Serr9/Ser32), respectively. The activated $\mathrm{NF}-\kappa \mathrm{B}$ dimer enters into the nucleus and regulates the transcription of several inflammation-related target genes. As a result, inflammatory reactions, apoptosis, free radical damage and other pathophysiological processes are mediated $(26,27)$. Previous studies have confirmed that activation of the $\mathrm{NF}-\kappa \mathrm{B}$ pathway is not only regulated by its inhibitor $\mathrm{I} \kappa \mathrm{B}$, but also by p38 MAPK, which further contributes to the transcription of inflammation-related genes (28). The present study indicated that downregulated PVT1 can markedly inhibit the MAPK/NF-kB pathway, thus being involved in the regulation of inflammatory factors. In the present study, the specific role of PVT1 in myocardial diseases was examined, and it was found that PVT1 was important in the occurrence and development of sepsis-induced inflammation and cardiac dysfunction. However, there were a number of limitations in the present study. Neither the expression of PVT1 in other tissues nor changes of local cytokines in the myocardium were measured in the present study. In addition, further investigation of the location of the expression of mRNA-143 in the heart is warranted.

In conclusion, PVT1 was upregulated in the myocardial tissues of sepsis rats, which inhibited cardiac function and promoted the secretion of inflammatory factors. The mechanism was found to be associated with the MAPK/NF- $\kappa \mathrm{B}$ pathway.

\section{Acknowledgements}

Not applicable.

\section{Funding}

No funding was received.

\section{Availability of data and materials}

All data generated or analyzed in the present study are included in this article.

\section{Authors' contributions}

FF and YQ designed the study and performed the experiments; FF, CD and CY established the animal models; YQ and CD collected the data; YQ and CY analyzed the data; FF prepared the manuscript. All authors have read and approved the final manuscript.

\section{Ethics approval and consent to participate}

The present study was approved by the Animal Ethics Committee of Lanzhou University Animal Center (Lanzhou, China).

\section{Patient consent for publication}

Not applicable.

\section{Competing interests}

The authors declare that they have no competing interests.

\section{References}

1. Liu V, Escobar GJ, Greene JD, Soule J, Whippy A, Angus DC and Iwashyna TJ: Hospital deaths in patients with sepsis from 2 independent cohorts. JAMA 312: 90-92, 2014.

2. Zanotti-Cavazzoni SL and Hollenberg SM: Cardiac dysfunction in severe sepsis and septic shock. Curr Opin Crit Care 15: 392-397, 2009.

3. Lodha R, Arun S, Vivekanandhan S, Kohli U and Kabra SK: Myocardial cell injury is common in children with septic shock. Acta Paediatr 98: 478-481, 2009.

4. Feng Y, Zou L, Zhang M, Li Y, Chen C and Chao W: MyD88 and Trif signaling play distinct roles in cardiac dysfunction and mortality during endotoxin shock and polymicrobial sepsis. Anesthesiology 115: 555-567, 2011.

5. Duncan DJ, Yang Z, Hopkins PM, Steele DS and Harrison SM: TNF-alpha and IL-1beta increase Ca2+ leak from the sarcoplasmic reticulum and susceptibility to arrhythmia in rat ventricular myocytes. Cell Calcium 47: 378-386, 2010.

6. Quinn JJ and Chang HY: Unique features of long non-coding RNA biogenesis and function. Nat Rev Genet 17: 47-62, 2016.

7. Wu H, Yang L and Chen LL: The diversity of long noncoding RNAs and their generation. Trends Genet 33: 540-552, 2017.

8. Gerstein MB, Bruce C, Rozowsky JS, Zheng D, Du J, Korbel JO, Emanuelsson O, Zhang ZD, Weissman S and Snyder M: What is a gene, post-ENCODE? History and updated definition. Genome Res 17: 669-681, 2007.

9. Hanson RL, Craig DW, Millis MP, Yeatts KA, Kobes S, Pearson JV, Lee AM, Knowler WC, Nelson RG and Wolford JK: Identification of PVT1 as a candidate gene for end-stage renal disease in type 2 diabetes using a pooling-based genome-wide single nucleotide polymorphism association study. Diabetes 56: 975-983, 2007.

10. Birnbaum S, Ludwig KU, ReutterH,Herms S, Steffens M, Rubini M, Baluardo C, Ferrian M, Almeida de Assis N, Alblas MA, et al: Key susceptibility locus for nonsyndromic cleft lip with or without cleft palate on chromosome 8q24. Nat Genet 41: 473-477, 2009.

11. Wang N, Liu X, Zheng X, Cao H, Wei G, Zhu Y, Fan S, Zhou H and Zheng J. Ulinastatin is a novel candidate drug for sepsis and secondary acute lung injury, evidence from an optimized CLP rat model. Int Immunopharmacol 17: 799-807, 2013.

12. Livak KJ and Schmittgen TD: Analysis of relative gene expression data using real-time quantitative PCR and the 2(-Delta Delta C(T)) method. Methods 25: 402-408, 2001.

13. Vincent JL and Marshall JC: Surviving sepsis: A guide to the guidelines. Crit Care 12: 162, 2008.

14. Hobai IA, Edgecomb J, LaBarge K and Colucci WS: Dysregulation of intracellular calcium transporters in animal models of sepsis-induced cardiomyopathy. Shock 43: 3-15, 2015.

15. Tessier JP, Thurner B, Jüngling E, Lückhoff A and Fischer Y: Impairment of glucose metabolism in hearts from rats treated with endotoxin. Cardiovasc Res 60: 119-130, 2003.

16. Fallach R, Shainberg A, Avlas O, Fainblut M, Chepurko Y, Porat E and Hochhauser E: Cardiomyocyte Toll-like receptor 4 is involved in heart dysfunction following septic shock or myocardial ischemia. J Mol Cell Cardiol 48: 1236-1244, 2010.

17. Horton JW, Maass D, White J and Sanders B: Nitric oxide modulation of TNF-alpha-induced cardiac contractile dysfunction is concentration dependent. Am J Physiol Heart Circ Physiol 278: H1955-H1965, 2000.

18. Eichenholz PW, Eichacker PQ, Hoffman WD, Banks SM, Parrillo JE, Danner RL and Natanson C: Tumor necrosis factor challenges in canines: Patterns of cardiovascular dysfunction. Am J Physiol 263: H668-H675, 1992.

19. Abraham E, Anzueto A, Gutierrez G, Tessler S, San Pedro G, Wunderink R, Dal Nogare A, Nasraway S, Berman S, Cooney R, et al: Double-blind randomised controlled trial of monoclonal antibody to human tumour necrosis factor in treatment of septic shock. NORASEPT II study group. Lancet 351: 929-933, 1998.

20. Damas P, Ledoux D, Nys M, Vrindts Y, De Groote D, Franchimont $\mathrm{P}$ and Lamy M: Cytokine serum level during severe sepsis in human IL-6 as a marker of severity. Ann Surg 215: 356-362, 1992. 
21. Niederbichler AD, Hoesel LM, Westfall MV, Gao H,Ipaktchi KR, Sun L, Zetoune FS, Su GL, Arbabi S, Sarma JV, et al: An essential role for complement $\mathrm{C} 5 \mathrm{a}$ in the pathogenesis of septic cardiac dysfunction. J Exp Med 203: 53-61, 2006.

22. Schulz R, Nava E and Moncada S: Induction and potential biological relevance of a $\mathrm{Ca}(2+)$-independent nitric oxide synthase in the myocardium. Br J Pharmacol 105: 575-580, 1992.

23. Kim EK and Choi EJ: Pathological roles of MAPK signaling pathways in human diseases. Biochim Biophys Acta 1802: 396-405, 2010.

24. Ridder DA and Schwaninger M: NF-kappaB signaling in cerebral ischemia. Neuroscience 158: 995-1006, 2009.

25. Zhang F, Li N, Jiang L, Chen L and Huang M: Neuroprotective effects of (-)-Epigallocatechin-3-Gallate against focal cerebral ischemia/reperfusion injury in rats through attenuation of inflammation. Neurochem Res 40: 1691-1698, 2015.
26. Wang X, Hu D, Zhang L, Lian G, Zhao S, Wang C, Yin J, Wu C and Yang J: Gomisin a inhibits lipopolysaccharide-induced inflammatory responses in N9 microglia via blocking the NF-kappaB/MAPKs pathway. Food Chem Toxicol 63: 119-127, 2014.

27. Raza SS, Khan MM, Ahmad A, Ashafaq M, Islam F, Wagner AP, Safhi MM and Islam F: Neuroprotective effect of naringenin is mediated through suppression of NF-kappaB signaling pathway in experimental stroke. Neuroscience 230: 157-171, 2013.

28. Dhawan P and Richmond A: A novel NF-kappa B-inducing kinase-MAPK signaling pathway up-regulates NF-kappa B activity in melanoma cells. J Biol Chem 277: 7920-7928, 2002.

(i) () () This work is licensed under a Creative Commons Attribution-NonCommercial-NoDerivatives 4.0 International (CC BY-NC-ND 4.0) License. 